\title{
Gitta Ho, George Grosz und Frankreich
}

\section{Catherine Wermester}

\section{OpenEdition \\ Journals}

Édition électronique

URL : http://journals.openedition.org/ifha/8982

DOI : $10.4000 /$ ifha. 8982

ISSN : 2198-8943

Éditeur

IFRA - Institut franco-allemand (sciences historiques et sociales)

\section{Référence électronique}

Catherine Wermester, « Gitta Ho, George Grosz und Frankreich », Revue de I'IFHA [En ligne], Date de recension, mis en ligne le, consulté le 23 septembre 2020. URL : http://journals.openedition.org/ifha/ 8982 ; DOI : https://doi.org/10.4000/ifha.8982

Ce document a été généré automatiquement le 23 septembre 2020.

(CIFHA 


\section{Gitta Ho, George Grosz und Frankreich}

\section{Catherine Wermester}

\section{RÉFÉRENCE}

Gitta Ho, George Grosz und Frankreich, Berlin: Reimer Verlag, 2016, 280 p., $49 €$ 
On savait que George Grosz s'était à plusieurs reprises rendu en France entre 1913 et 1928 mais, jusqu'ici, aucune étude approfondie n'avait été consacrée à ces séjours, à leurs conséquences sur la carrière de l'artiste, ni aux motivations de Grosz lui-même. Tiré d'une thèse soutenue à Hambourg en 2011, l'ouvrage de Gitta Ho comble amplement cette lacune. On y découvre, étayée par la lecture et le dépouillement soigneux d'archives, de correspondances ou encore de revues, une patiente reconstruction des semaines passées en France par l'artiste. À Paris bien sûr, qui peut encore se prévaloir à l'époque du titre glorieux de capitale mondiale de l'art, mais aussi en Bretagne ou à Marseille - lieux de villégiature qu'il apprécie -, à Nice ou en

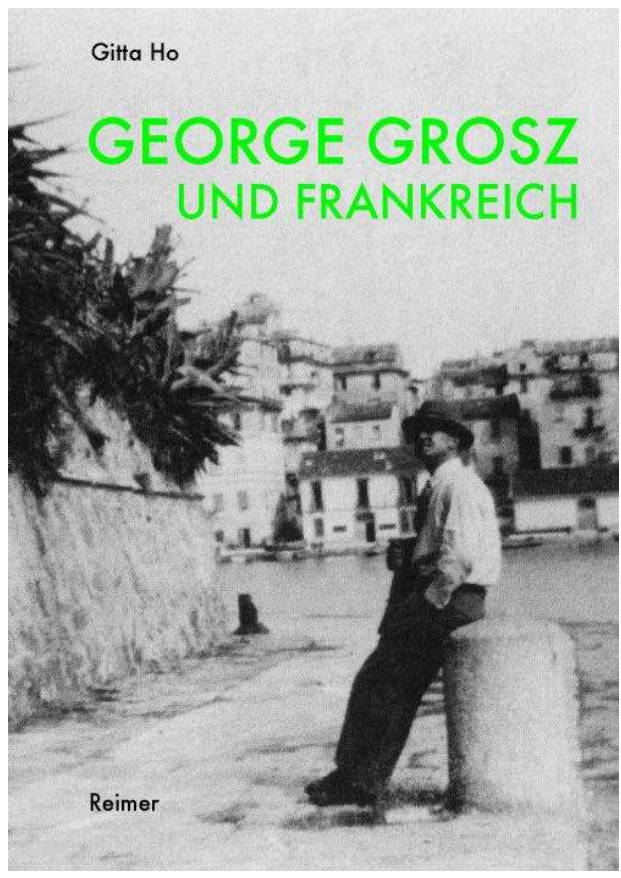
Corse, dont il conservera un souvenir plus que mitigé.

L'art français le plus récent, le jeune Grosz le découvre avant 1914, à Berlin, grâce aux marchands parisiens qui, comme dans tous les centres européens, y déploient une grande activité, et grâce à des galeries telles que la célèbre Sturm de Herwath Walden, acteur déterminant dans les échanges franco-allemands avant la guerre. De même, c'est en 1913 que l'artiste effectue son premier voyage à Paris. Dans les années 1920, ses écrits témoignent non seulement de sa connaissance, mais encore de son intérêt pour l'art et la littérature français ; quant à son œuvre, elle ne dit pas autre chose.

En démontrant cette attention particulière et en exemplifiant la façon dont Grosz regarde et intègre à sa propre recherche le cubisme ou encore l'art de Delaunay, G. Ho entend révoquer en doute les déclarations tonitruantes de l'artiste contre l'art français et la France au début de la décennie. Ainsi que le rappelle l'auteure, il existait chez bon nombre d'artistes allemands de l'époque un ressentiment plus ou moins marqué contre la position dominante de la France, laquelle se manifestait aussi sur le marché allemand, encourageant galeristes et critiques en vue à prendre fait et cause pour l'art français, au détriment, affirmaient d'aucuns, de l'art allemand. Grosz pour sa part, et ainsi que le rapporte l'auteure à plusieurs reprises, n'entrait pas dans ce genre de débats, et encore moins dans les querelles nationalistes. Sans doute faut-il penser que son rejet de la France et de l'art français obéissait à un impératif stratégique. Parce qu'ils occupaient de fait une position dominante dans le marché de l'art et la production théorique, ils apparaissaient comme le parangon d'une économie artistique et d'une définition sociale de l'art que l'artiste entendait alors contester, sans s'embarrasser de nuances.

Dans son travail formel, il en allait tout autrement. À cet égard, et tout au long de son ouvrage, l'auteure ajoute aux faits, aux rencontres et aux déclarations vérifiables, des hypothèses et des interprétations convaincantes. Ainsi de son rapprochement entre $\grave{A}$ la Russie, aux ânes et aux autres (1911) de Marc Chagall, et John l'assassin de femmes peint 
par Grosz en 1918. Inédite et bien menée, la comparaison constitue en outre un exemple pertinent à partir duquel réfléchir aux modes de réappropriation sensibles dans l'œuvre de l'artiste en général. Par ailleurs, si c'est aussi un intérêt bien compris qui, pour partie, fonde le désir de Grosz de se faire connaître et reconnaître dans la capitale de l'art comme beaucoup d'autres l'avaient essayé avant lui, il y réussit parfaitement, devenant même après 1924, date de sa première exposition personnelle parisienne organisée à la galerie Billiet, l'artiste allemand contemporain le plus célèbre en France.

G. Ho expose de façon circonstanciée les conditions de ce parcours. Ainsi montre-t-elle comment l'artiste a pu compter sur de nombreux appuis et médiateurs avec leurs réseaux de sociabilité, parmi lesquels les artistes Jules Pascin ou Frans Masereel et les écrivains Yvan Goll ou Pierre Mac Orlan. Ce dernier parlait à propos des dessins de Grosz de "fantastique social », notion aux contours plutôt vagues qu'il utilisait par ailleurs pour rendre compte des aspects de la modernité qui lui paraissaient obscurs et mystérieux. Grosz s'en était d'abord irrité, soupçonnant derrière cette expression un refus de prendre en compte la dimension explicitement marxiste de ses travaux. Ainsi que le rapporte G. Ho, discuter avec l'écrivain français avait cependant convaincu l'artiste qu'il n'avait pas affaire à un « antibolcheviste ».

De Grosz artiste engagé, il est beaucoup question et, de fait, les cercles qui le soutiennent et le font connaître partagent souvent tout ou partie de ses positions. Les ayant identifiées, G. Ho montre aussi à quels malentendus et à quels préjugés le dessinateur politique allemand a été confronté. Ainsi, ses dessins satiriques, clairement orientés contre les gouvernements de la République de Weimar mais aussi, au-delà d'eux, contre le militarisme et les relations de domination, sont facilement perçus dans la France de l'entre-deux-guerres comme hostiles à l'Allemagne. Dans le même temps cependant, et ainsi que l'établit l'auteure, les critiques associent moins Grosz à telle ou telle tendance de l'art qu'aux cercles engagés qu'il fréquente et qui assurent au premier chef la diffusion de son œuvre. Parmi eux, celui de Clarté, revue d'intellectuels qu'un groupe de communistes entend constituer en centre d'éducation révolutionnaire international.

La fin de la décennie s'accompagne d'une réévaluation critique de Grosz et de son œuvre. Peu à peu, l'artiste engagé et / ou anti allemand tend à s'effacer derrière la figure de l'artiste allemand et expressionniste. Mais l'auteure s'intéresse aussi aux discours que Grosz lui-même tient à divers moments de sa vie sur ces dessins réputés politiques. Parfois volontairement outrés et volontiers déroutants, ils complètent et tantôt rectifient les termes de sa réception en France et, s'agissant de la compréhension générale de son œuvre, tendent à la complexifier. Ainsi G. Ho rapporte-t-elle les déclarations faites par l'artiste dans les années 1950 aux États-Unis, où il s'est installé en 1932. En pleine période maccarthyste, Grosz y évoque un prétendu piratage partisan de ses dessins à des fins de propagande communiste. Sans trancher la question, condamnée à rester plus ou moins ouverte, du degré d'engagement de l'artiste dans l'idéal communiste, l'auteure s'attache à mettre en perspective les affirmations et les revirements successifs de Grosz à cet égard.

Les développements consacrés aux séjours à caractère plus touristique de l'artiste et de sa famille en France, loin de Paris, ne sont pas moins intéressants. G. Ho montre ainsi comment la peinture de paysage et le portrait auxquels il s'adonne après 1925 font, dans son œuvre, contrepoint à ses dessins politiques. La peinture de paysage en 
particulier, vers laquelle le pousse son marchand Alfred Flechtheim, l'exhortant à s'éloigner de son travail d'artiste engagé pour enfin peindre de "belles choses », est tantôt présentée par Grosz comme une source de revenus, tantôt comme un exercice de peinture et de composition, ce que l'auteure démontre par ailleurs, analyses à l'appui. En Allemagne, il se trouve des critiques pour se réjouir que les séjours de Grosz en France l'aient, selon eux, éloigné de l'art politique. En France, le critique René Jean est l'un de ceux qui se prennent à espérer que, sans cesser d'être allemand, Grosz puisse atteindre à l'harmonie, l'équilibre et la sérénité réputés caractéristiques de la France et de l'art français...

À propos de la réception de l'art allemand en France, on rappellera les travaux incontournables de Mathilde Arnoux (Les Musées français et la peinture allemande, 1871-1981) et de Marie Gispert ("L'Allemagne n'a pas de peintre ». Diffusion et réception de l'art allemand moderne en France, 1918-1939), deux études d'ensemble témoignant toutes deux de l'importance des enquêtes de détail en la matière. À cet égard, le George Grosz und Frankreich de G. Ho convainc s'il en était encore besoin que l'idée longtemps dominante d'un rejet unanime de l'art allemand dans la France de l'entre-deux-guerres doit céder la place à un portrait nuancé. Contribution à ce grand domaine de recherche, l'ouvrage de G. Ho, documenté sans jamais être d'une lecture fastidieuse, enrichit aussi les connaissances à propos de George Grosz lui-même.

\section{INDEX}

Index chronologique : Époque contemporaine

Thèmes : Histoire de la culture, Histoire de l'art 